\title{
Direct laser writing: biomimetic photonics and superresolution nanolithography (Presentation Video)
}

\section{Min Gu}

Min Gu, "Direct laser writing: biomimetic photonics and superresolution nanolithography (Presentation Video)," Proc. SPIE 8954, Nanoscale Imaging, Sensing, and Actuation for Biomedical Applications XI, 895415 (11 March 2014); doi: $10.1117 / 12.2064967$

SPIE. Event: SPIE BiOS, 2014, San Francisco, California, United States 


\section{Direct laser writing: biomimetic photonics and superresolution nanolithography (Presentation Video)}

Min Gu, Swinburne Univ. (Australia)

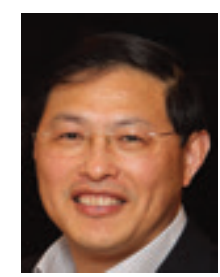

ABSTRACT

The video for this presentation is unavailable. 